\title{
Solution-processable electrode-material embedding in dynamically inscribed nanopatterns (SPEEDIN) for continuous fabrication of durable flexible devices
}

\author{
Wonseok Lee $\mathbb{D}^{1}$, Hyoungseok Chae ${ }^{1}$, Dong Kyo Oh $\mathbb{D}^{2}$, Minyoung Lee ${ }^{1}$, Hyunsoo Chun ${ }^{3}$, Gyubeom Yeon ${ }^{1}$, \\ Jaewon Park', Joohoon Kim $\mathbb{D}^{2}$, Hongseok Youn ${ }^{4}$, Junsuk Rho $\mathbb{E}^{2,5,6 凶}$ and Jong G. Ok $\mathbb{D}^{1 凶}$
}

\begin{abstract}
A facile and scalable lithography-free fabrication technique, named solution-processable electrode-material embedding in dynamically inscribed nanopatterns (SPEEDIN), is developed to produce highly durable electronics. SPEEDIN uniquely utilizes a single continuous flow-line manufacturing process comprised of dynamic nanoinscribing and metal nanoparticle solution coating with selective embedding. Nano- and/or micro-trenches are inscribed into arbitrary polymers, and then an Ag nanoparticle solution is dispersed, soft-baked, doctor-bladed, and hard-baked to embed Ag micro- and nanowire structures into the trenches. Compared to lithographically embossed metal structures, the embedded SPEEDIN architectures can achieve higher durability with comparable optical and electrical properties and are robust and power-efficient even under extreme stresses such as scratching and bending. As one tangible application of SPEEDIN, we demonstrate a flexible metal electrode that can operate at $5 \mathrm{~V}$ at temperatures up to $300^{\circ} \mathrm{C}$ even under the influence of harsh external stimuli. SPEEDIN can be applied to the scalable fabrication of diverse flexible devices that are reliable for heavy-duty operation in harsh environments involving high temperatures, mechanical deformations, and chemical hazards.
\end{abstract}

\section{Introduction}

Metal nanostructures, including nanoporous metal films, self-assembled metal layers, and micro- and nanopatterned metal films, have inspired many diverse functional applications in photonics, plasmonics, and electronics $^{1-5}$. Among these structures, metal micro- and nanopattern structures (MNPs) have provided highly accessible and widely applicable frameworks for most

\footnotetext{
Correspondence: Junsuk Rho (jsrho@postech.ac.kr) or

Jong G. Ok (jgok@seoultech.ac.kr)

'Department of Mechanical and Automotive Engineering, Seoul National University of Science and Technology, Seoul 01811, Republic of Korea

${ }^{2}$ Department of Mechanical Engineering, Pohang University of Science and Technology (POSTECH), Pohang 37673, Republic of Korea

Full list of author information is available at the end of the article These authors contributed equally: Wonseok Lee, Hyoungseok Chae, Dong Kyo Oh
}

practical devices, including transparent electrodes, sensor platforms, and plasmonic templates ${ }^{5-10}$. For instance, silver (Ag) has been one of the most versatile MNP materials owing to its excellent mechanical malleability, robust electrical and thermal conductivity, and unique plasmonic and photonic characteristics ${ }^{11-16}$.

The fabrication of MNPs typically involves three major steps of metal deposition, lithographic patterning, and etching ${ }^{17,18}$. Common protocols for each step have been well established for years, such as by relying on physical vacuum deposition, photo-, e-beam, and nanoimprint lithography, wet and dry etching, and so on ${ }^{19-23}$. For example, sputtering is a widely used physical vapor deposition, which can manipulate optical reflectivity in the UV/Vis range with the deposited nanoparticle size, distance, shape, and interface morphology ${ }^{24,25}$. While

\section{(c) The Author(s) 2021}

(c) (i) Open Access This article is licensed under a Creative Commons Attribution 4.0 International License, which permits use, sharing, adaptation, distribution and reproduction cc) in any medium or format, as long as you give appropriate credit to the original author(s) and the source, provide a link to the Creative Commons license, and indicate if changes were made. The images or other third party material in this article are included in the article's Creative Commons license, unless indicated otherwise in a credit line to the material. If material is not included in the article's Creative Commons license and your intended use is not permitted by statutory regulation or exceeds the permitted use, you will need to obtain permission directly from the copyright holder. To view a copy of this license, visit http://creativecommons.org/licenses/by/4.0/. 
effective, these conventional methods are often challenged by the increasing demand for MNPs due to their intrinsic limits on cost, time, and $\operatorname{area}^{26}$. To overcome these comprehensive problems, alternative nanofabrication techniques have been introduced ${ }^{27-32}$. However, the needs of high-temperature vacuum processing and/or plasma/chemical etching can still restrict the applicable materials, especially for flexible and organic-based devices $^{33,34}$. In addition, such a lithographically defined MNP is typical of an 'embossed' structure on the substrate surface, which is prone to be damaged over time by external irritations, such as scratches and hard contacts, or internal degradations, such as cracks and delaminations $^{35-37}$.

In these regards, a more productive and scalable methodology is needed for a facile and cost-effective fabrication of more durable MNPs on desirable flexible substrates $^{38-41}$. More specifically, the proposed method may realize photomask- and etch-free micro- and nanopatterning and vacuum-free metalizing therein at a low temperature $^{42-45}$. To this end, a solution-processable electrode-material embedding in dynamically inscribed nanopatterns, named SPEEDIN, is developed in this study. First, in SPEEDIN, a micro- or nanopatterned trench structure is continuously created on flexible polymers, such as polyimide (PI) substrate, with high mechanical and resistant chemical properties by dynamic nanoinscribing (DNI) ${ }^{46,47}$. In DNI, an edge of a patterning mold continuously inscribes the seamless micro- or nanograting pattern on various flexible substrates through a linear stroke under conformal contact, but there is no systematic DNI application on PI due to its tough machinability; also, multiple DNI strokes can be sequentially combined to readily create multidimensional patterns on PI. Then, a colloidal Ag nanoparticle solution is simultaneously coated on and doctor-bladed off the trench-patterned surface, which is annealed by slight baking to embed the Ag wires selectively inside trenches.

SPEEDIN uniquely enables the continuous and highspeed embedding of highly durable metal structures in scalable micro- and nanopatterns without resorting to masked lithography, vacuum deposition, or additional etching. Overcoming the limits and drawbacks of conventional embossed MNPs vulnerable to physical damages, SPEEDIN can achieve the highly durable metal frameworks generally applicable to heavy-duty flexible devices. As one vivid example, a flexible device that can be reliably heated up to $300{ }^{\circ} \mathrm{C}$, even under harsh environments involving scratching, tension, bending, and twisting, is demonstrated.

\section{Results and discussion}

The SPEEDIN procedure comprises three main steps: DNI patterning, metal solution coating, and doctorblading, all of which can be performed in a continuous and high-speed fashion $(\sim 10 \mathrm{~mm} / \mathrm{s})$ without timeconsuming lithography and vacuum aids, thus making it configurable as a single flow-line manufacturing system. Figure 1a schematically illustrates such a continuous, single-stroke principle of SPEEDIN. First, a well-cleaved Si ridge mold edge $(\sim 2 \mu \mathrm{m}$ linewidth, $5 \mu \mathrm{m}$ height, and $50 \mu \mathrm{m}$ period, unless otherwise noted; Fig. 1b) slides on a flexible substrate under conformal contact (with a typical force of $1-5 \mathrm{~N}$ ) and proper heating (typically near the

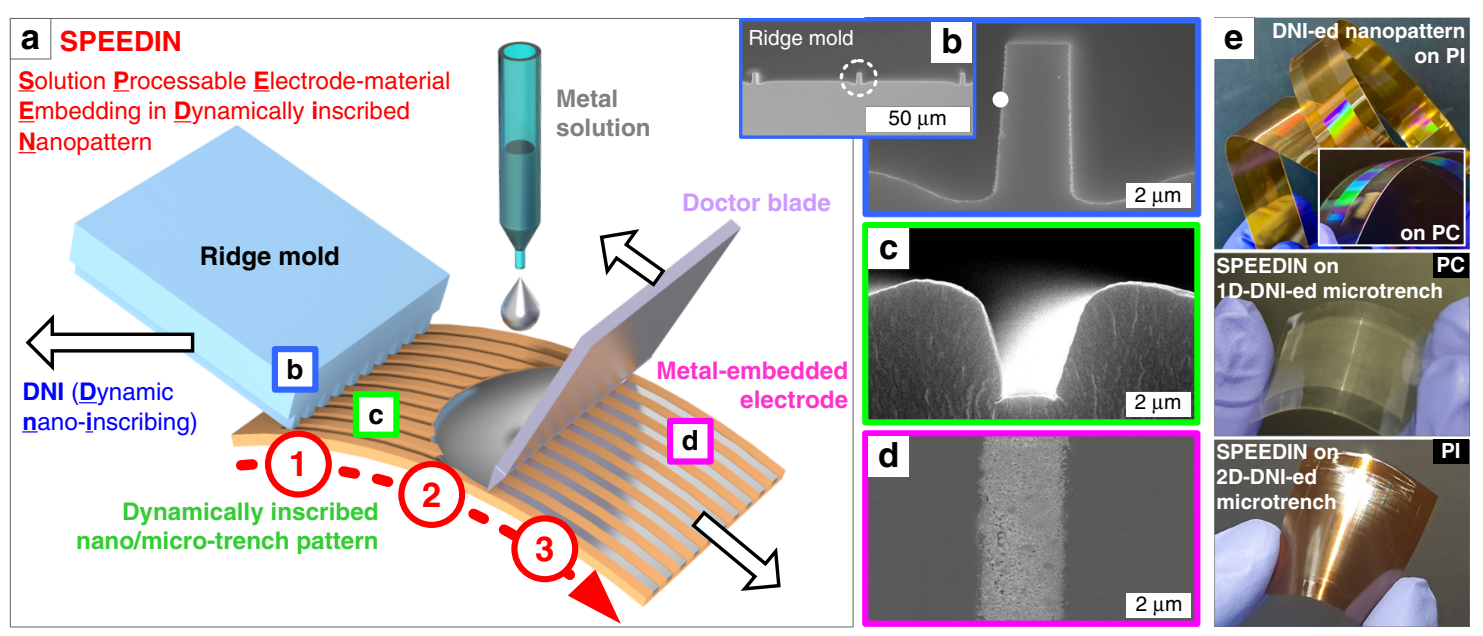

Fig. 1 Solution-processable electrode-material embedding in dynamically inscribed nanopatterns (SPEEDIN). a Schematic illustration of the SPEEDIN process comprising three sequential processes: (1) DNI, (2) metal solution coating, and (3) doctor-blading. The microridge mold (b) can continuously inscribe the (c) microtrench where (d) the metal microwire can be selectively embedded by controlled coating, baking, and doctorblading. e Examples of the seamless large-area nanopatterns continuously DNI-ed on PI (top) and PC (top inset), and the SPEEDIN-ed sample on flexible PC (middle) and PI (bottom) substrates 
glass transition temperature $\left(T_{g}\right)$ of the substrate material). Here, the substrate surface undergoes continuous plastic deformation to the trench pattern (Fig. 1c) without surface degradation by the continuous inscription of the ridges in a mold edge. ${ }^{46}$ The substrate materials employed in this DNI patterning include polycarbonate (PC) and PI, which can be seamlessly patterned over large areas as exhibited in Fig. 1e. While the patterning depth and profile can be tailored with a critical resolution of an $\sim 50 \mathrm{~nm}$ period and an $\sim 1: 1$ aspect ratio by controlling the inscribing force, temperature, and speed, as systematically demonstrated elsewhere ${ }^{46}$. The DNI patterning temperatures for PC and PI were controlled to near their respective $T_{g}$ 's of $150^{\circ} \mathrm{C}$ and $310^{\circ} \mathrm{C}$, with the contacting force set to $3 \mathrm{~N}$ in this study, to ensure consistent and faithful trench patterning. Notably, DNI can readily create scalable micro- and nanopatterns on the PI film that otherwise demands tricky etching work owing to its excellent mechanical and chemical durability, which makes it particularly promising for developing diverse 'heavy-duty' flexible MNP devices.

An aqueous colloidal solution containing Ag nanoparticles (average diameter $\sim 100 \mathrm{~nm}$ ) is then coated on the DNI-patterned surface, which is soft-baked, doctorbladed, and hard-baked to leave the solidified Ag wires embedded in the trenches as shown in Fig. 1d. Figure S1 depicts the exemplary SPEEDIN procedure. Here, the tactful arrangement of the Ag embedding sequence comprising soft- and hard-baking and doctor-blading is very important for successful SPEEDIN. Figure S2 in the Supplementary Information comparatively demonstrates several control arrangements of soft-baking, hard-baking, and doctor-blading sequences for the 1D-and 2D-DNI-ed microtrench pattern surfaces. If doctor-blading is applied to the as-coated, nonbaked Ag solution, most Ag, remaining liquid, is swept away with poor embedding into the DNI-ed trench. If doctor-blading is applied late after all baking is finalized, the already-solidified $\mathrm{Ag}$ layer cannot be cleanly peeled off the surface, leaving a spotty $\mathrm{Ag}$ residue behind. A soft-baking (typically at $100^{\circ} \mathrm{C}$ for $1 \mathrm{~min}$ ) approach can change the as-coated Ag solution to a viscous concentrate with reduced solvent but still keeps it in a liquid phase. Doctor-blading at this time can selectively scrape off the top surficial Ag coating while keeping the trench-embedded $\mathrm{Ag}$ in place. Hard-baking (typically at $120^{\circ} \mathrm{C}$ for $5 \mathrm{~min}$ ) is then applied to fully remove the residual solvent and complete the SPEEDIN structure.

An adequate initial Ag solution coating is another essential consideration for reliable SPEEDIN. Airbrushing may be one possible candidate for conformal and scalable coating of the Ag nanoparticle solution over the DNI-ed topographic surface. However, the uncontrolled solvent vaporization concurrently occurring during airbrushing can force the Ag nanoparticles to be spread and dried all over the surface. This can disturb the clean, selective scraping-off process, which is experimentally verified as shown in Fig. S3 in the Supplementary Information. Drop-casting may be another practical choice, especially for microscale trench patterns. However, dropping an excessive amount of the Ag solution can cause difficulty for uniform and time-efficient soft-baking and is prone to wasting the overflown Ag nanoparticles upon doctorblading. We could obtain an optimal thickness of Ag thin film by controlling the spin-coating speed depending on the microtrench width. For the typical 1-2 $\mu \mathrm{m}$-wide trenches shown in Fig. 1, a coating speed of $\sim 1000 \mathrm{rpm}$ is reasonable for the $\mathrm{Ag}$ solution used in this study; too fast a coating speed (e.g., $\sim 3000 \mathrm{rpm}$ ) could undesirably curtail the embedded Ag wire width due to insufficient initial coating (see Fig. S4 in the Supplementary Information for comparative results). For wider microtrench patterns, such as of an $\sim 10 \mu \mathrm{m}$ width, we can lower the coating speed (e.g., $\sim 500 \mathrm{rpm}$ ) to facilitate the Ag solution filling into the trenches. For nanoscale trench patterns, as the effect of capillary force becomes more pronounced, the drop-casting of a controlled amount of the Ag solution under gentle agitation can be a rational method. Several exemplary SPEEDIN structures fabricated on wider microtrench and nanotrench patterns are demonstrated in Fig. S5 in the Supplementary Information.

SPEEDIN can achieve the facile, scalable, and clean embedding of metal microwires in various polymer films. Figure 2 collectively demonstrates the SPEEDIN results for 1D- and 2D-DNI-ed microtrench patterns created on PI and PC substrates. The DNI patterning can be sequentially performed along multiple directions to create 1D, 2D, and further multidimensional micro- and nanopattern structures, as schematically illustrated in Fig. 2a. Notably for 2DDNI, after the first DNI stroke was made with the contacting force of $3 \mathrm{~N}$, the force during the second stroke was controlled to be $\sim 50 \%$ (i.e., $\sim 1.5 \mathrm{~N}$ ) in order to avoid excessive deformation of the first-stroked DNI pattern lines. This can secure the 'open crossing' structure for each intersection, which is favorable for mesh-type metal nanowire fabrication by subsequent $\mathrm{Ag}$ solution embedding (see the inset to Fig. 2e2). The comparative optical images taken before and after performing 1D- and 2D-SPEEDIN on PI and PC are shown in Fig. $2 \mathrm{~b}$ and c, respectively. The scanning electron microscopy (SEM) imaging for these structures (Fig. 2d and e; before and after SPEEDIN, respectively) confirms the clean, well-connected Ag microwire structures fabricated by SPEEDIN. The enlarged SEM images shown as the insets to Fig. 2e disclose that the continuous $\mathrm{Ag}$ lines are only inside the DNI-ed trenches, with very little Ag residue on the surrounding areas. This is also verified by energy-dispersive X-ray spectroscopy (EDX), as seen in Fig. S6 in the Supplementary Information. 

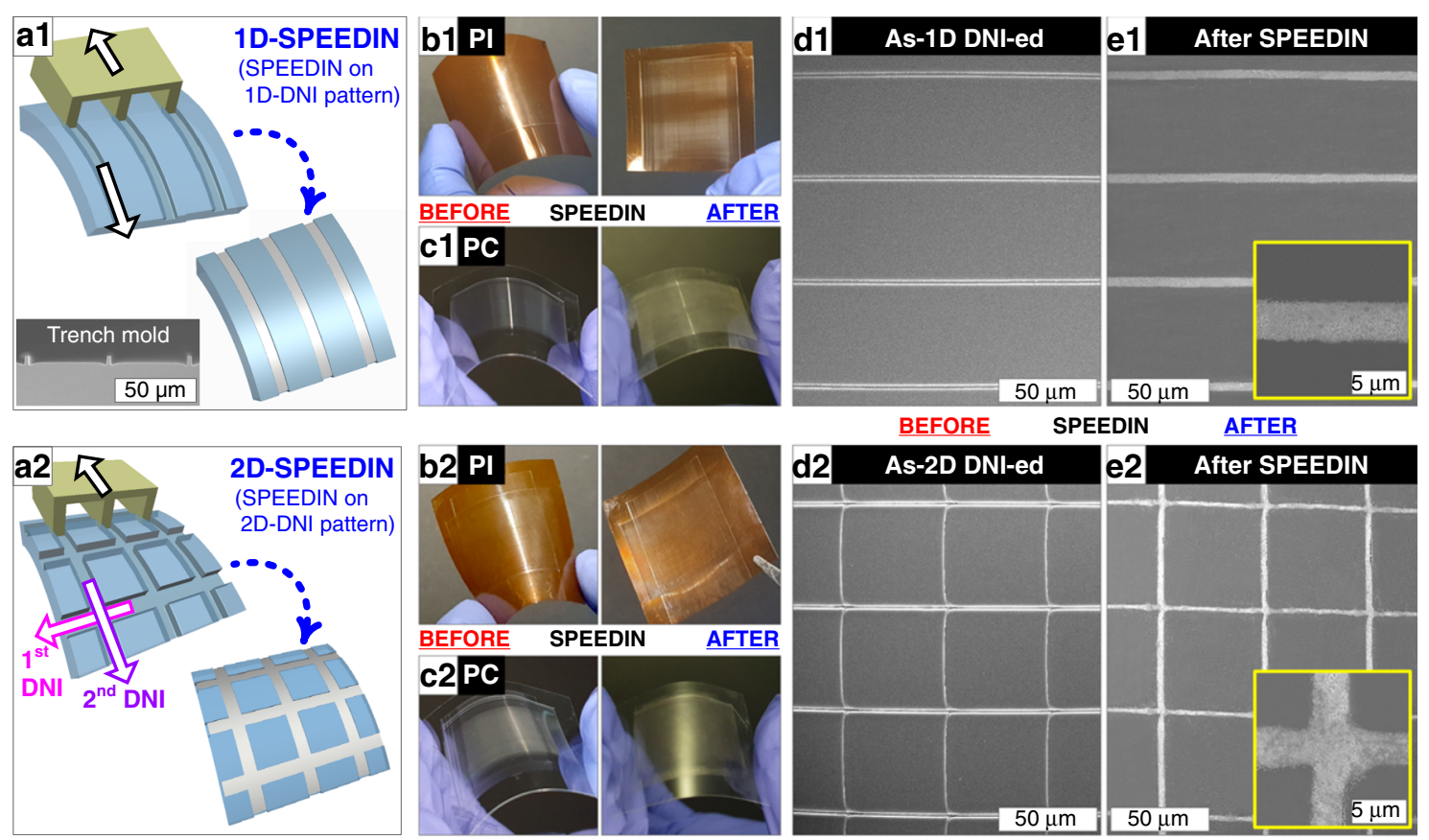

Fig. 2 Comparative demonstration of the 1D-SPEEDIN (top row) and 2D-SPEEDIN (bottom row). a illustrations of DNI schemes before and after Ag embedding, (b) as-DNI-ed (left) and SPEEDIN-ed (right) samples on PI, (c) as-DNI-ed (left) and SPEEDIN-ed (right) samples on PC, and SEM images of (d) as-DNI-ed and (e) SPEEDIN-ed structures. The insets to (e) show the enlarged views of embedded Ag microwires

The SPEEDIN-ed polymer films can possess reasonable optical transmittance as well as electrical conductivity, although the embedding of Ag structures in the transparent substrates may obviously call for some compensation. Figure 3a shows the measurement results for the optical transmittances of the 1D- and 2D-SPEEDIN-ed structures formed on PC substrates (with a measured reference transmittance of $\sim 90 \%$ with respect to air). Compared to the $\sim 10 \%$ transmittance measured for a continuous, nonpatterned Ag layer coated on bare PC film, the 1D- and 2D-SPEEDIN-ed Ag microstructures on PC films indicate transmittances of $\sim 30 \%$ and $\sim 40 \%$, respectively. The optical images of the real samples (Fig. 3b) demonstrate more vividly that these structures are transparent for clear transmission of the background images. The decrease in optical transmittance may be due to additional factors other than the Ag microwires' lightblocking, possibly involving intrinsic scattering and surface plasmon resonance from the sparsely remaining $\mathrm{Ag}$ nanoparticles. Such optical characteristics of SPEEDIN-ed MNPs might be similar to those studied for the 'embossed' Ag MNPs conventionally relying on the photolithography-and-then-etching fabrication proto$\mathrm{Col}^{48,49}$. Meanwhile, the sheet resistances for the 1D- and 2D-SPEEDIN structures fabricated on PI films were measured to be 26.3 and $19.8 \Omega / \mathrm{sq}$ on average (see Table S1 in the Supplementary Information for detailed measurement data).
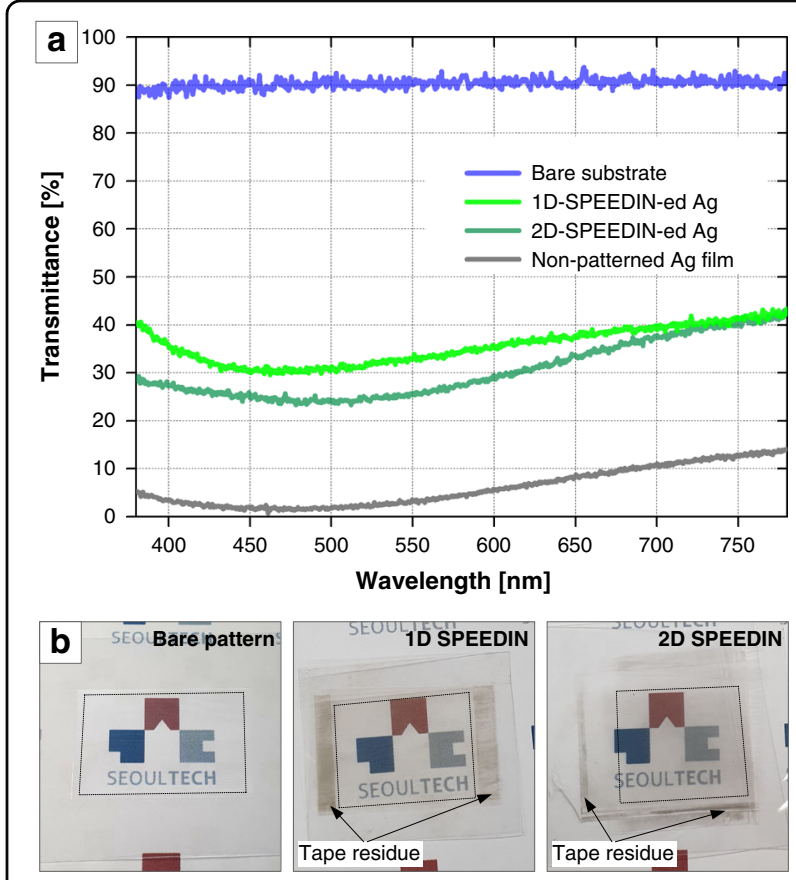

Fig. 3 Optical properties and photographs of SPEEDIN-ed sample. a Optical transmittances measured for bare, 1D-SPEEDIN-ed, 2D-SPEEDIN-ed, and nonpatterned Ag-coated PC samples. b Optical images of the fabricated samples. The dark areas surrounding the SPEEDIN-ed areas are generated by the tape residues used for sample mounting 


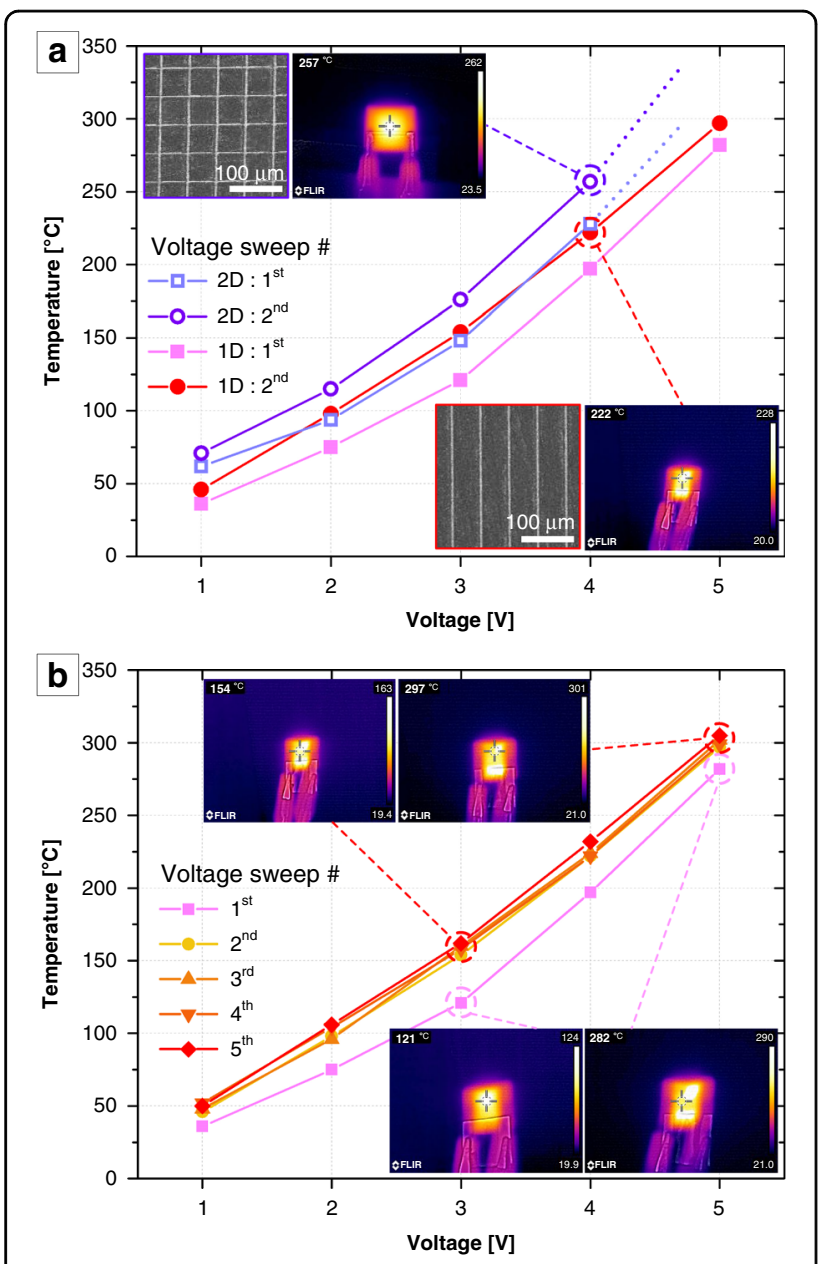

Fig. 4 Heating devices fabricated by SPEEDIN. a Heating performance of the 1D- and 2D-SPEEDIN-on-PI samples, comparatively plotting the temperatures measured for the first-round and secondround operations under a voltage sweep from 1 to $5 \mathrm{~V}$. The insets show the SEM images and representative IR camera images taken at the $4 \mathrm{~V}$ operation. $\mathbf{b}$ Five times repeated heating performance of the 1D-SPEEDIN-on-PI sample with the identical setup. The insets show the representative IR camera images taken at the 3 and $5 \mathrm{~V}$ operations

While these optical and electrical properties may be commonly practicable for transparent and flexible devices, the uncommon ability to the facile MNP embedding in mechanically and chemically robust substrates can allow the SPEEDIN structures to be utilized in numerous diverse applications by affording reliable operation in a harsh environment. For instance, the SPEEDIN-on-PI structures can be used as high-performance flexible and transparent joule-heaters that can achieve up to $\sim 300{ }^{\circ} \mathrm{C}$ heating at $5 \mathrm{~V}$ operation voltage. Figure 4 shows the heating performances of the $1 \mathrm{D}$ - and 2D-SPEEDIN-on-PI samples, characterized by plotting the IR camerameasured temperature versus sweep voltage (V). According to Joule's law of resistive heating: $P \sim V^{2} / R$ (where $R$ is resistance), the power of heating $P$ may be higher for more conductive electrode frameworks under the same $V$ operation. Consistently, the 2D-SPEEDIN-onPI structure can realize more power-efficient heating overall, as shown in Fig. 4a.

The cyclic operations by repeated voltage sweeps provide us with two further important findings. First, compared to the first-round heating using the asSPEEDIN-ed structures, the follow-up rounds' operations indicate generally improved heating efficiencies. As comparatively seen in the first- and second-round operations of the 1D- and 2D-SPEEDIN-on-PI samples (Fig. 4a), this was systematically examined through multiple times repeated operations of the $1 \mathrm{D}$ SPEEDIN-on-PI sample. Figure $4 \mathrm{~b}$ shows the heating performance for the first five-round operations, and the IR camera images taken for each measurement point are additionally provided in Fig. S7 in the Supplementary Information. Although not shown here, we additionally confirmed that the heating curve is stabilized from the initial operation, for instance, the next fifty times. We can comprehend such an interesting 'aging' characteristic by taking a closer look at the morphologies of the $\mathrm{Ag}$ wires before and after initial heating. Figure S8 comparatively shows the as-SPEEDIN-ed, never-heated Ag wire and the Ag wire 'annealed' with initial heating experience, along with the schematic illustrations of respective cross-sections. Initially, the as-SPEEDIN-ed Ag nanoparticles are loosely packed with nanoporous interspaces, resulting in higher contact resistance. Once these Ag nanoparticles undergo the heating cycle (i.e., thermal annealing ${ }^{50,51}$ ), they are more closely packed by merging into larger aggregations with reduced pores, as also supported by the contracted linewidth observed in the top-view SEM image. This densification of Ag nanoparticles can lead to a decrease in contact resistance and enhanced resistive joule-heating for the follow-up operations.

Another crucial point discovered from the cyclic operation is that the SPEEDIN-ed structures exhibit highly consistent and reproducible heating performance upon repeated heating cycles. It is normally considered that most of the MNP-embossed and/or metallic thinfilm-deposited resistive heating architectures suffer from electrode cracking and/or delamination during the repeated operations. By resolving that issue, the stable and steady cyclic operation investigated in the SPEEDINbased heating architectures (Fig. 4) may be attributed to the enhanced adhesion between the embedded metal microwires and substrate trenches over the increased interfacial surface areas (see the inset diagrams in Fig. 5a). In addition to the robustness against those internal degradations, the SPEEDIN architectures, inherently comprising embedded wires, can provide excellent durability against external irritations such as scratches and 


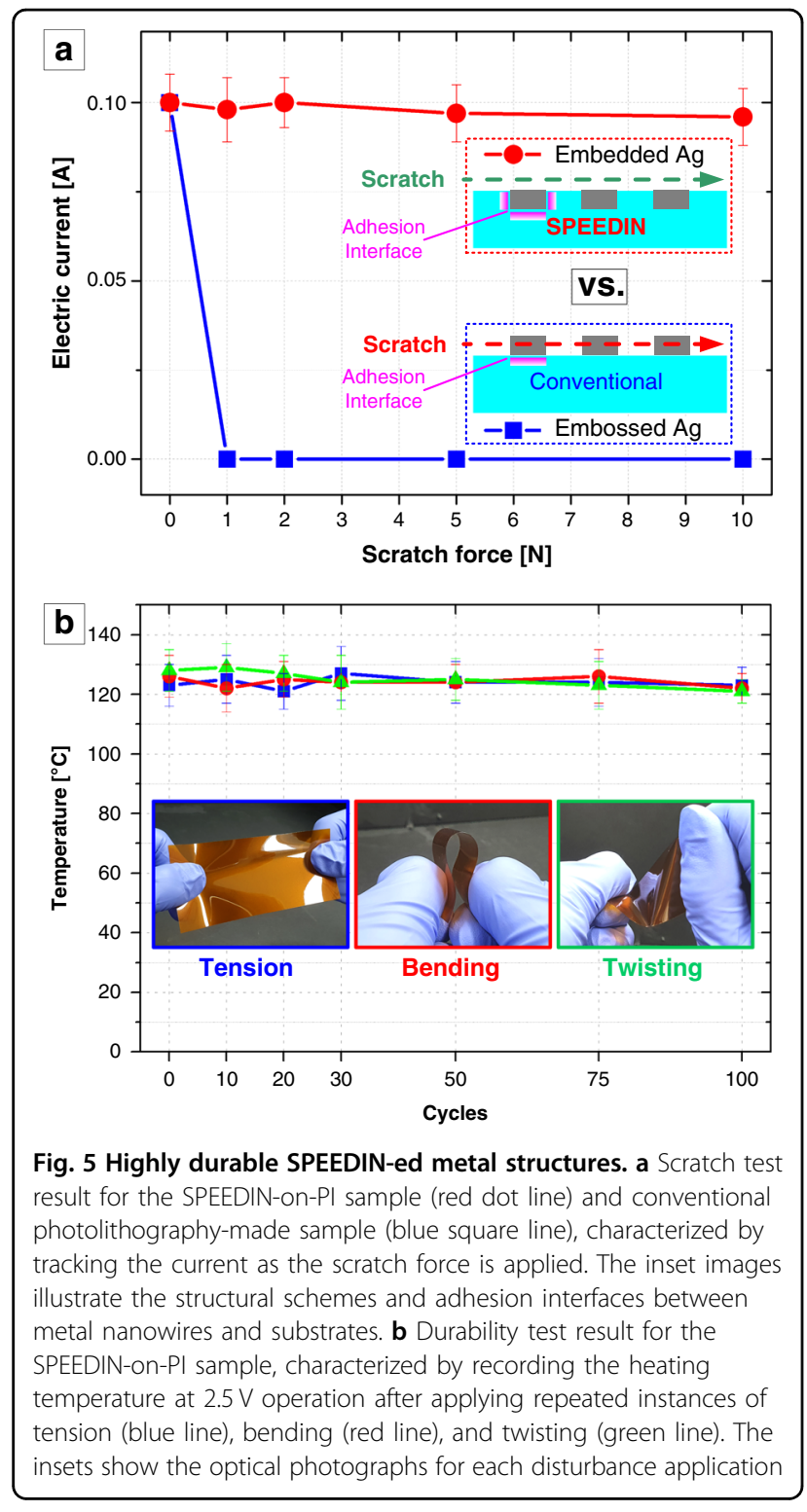

hard contacts. Here, we conducted a simple scratch test for two types of MNP electrodes with similar wire geometries but fabricated through the different protocols: SPEEDIN and photolithography etching. Once each of the fabricated MNP samples was put on the scale and was initially set to an $\sim 0.1 \mathrm{~A}$ current operation, a tweezer scratch was applied with a controlled force (read by the scale). The experimental detail of this test is described in the Experimental Section, along with the scheme in Fig. S9 in the Supplementary Information. Figure 5a shows the scratch test results for the embedded SPEEDIN and the conventional embossed MNP electrodes, characterized by plotting the electrical current versus scratch force. It can be clearly seen that the SPEEDIN-ed electrode sustains steady operation against the scratch applied with up to an $\sim 10 \mathrm{~N}$ force, whereas the embossed MNP electrode is shortly disconnected right after the $1 \mathrm{~N}$ scratch. Although the SPEEDIN structure is sustainable beyond the $\sim 10 \mathrm{~N}$ scratch, a deformation of the substrate material, such as tearing and crumpling, occurs rather than the destruction of the electrode itself. While quite simplified, this scratch test exemplifies the durability of the SPEEDIN electrodes against external mechanical stimulation compared to fragile conventional MNPs.

The durable and steadfast functioning of the SPEEDIN architecture in a highly reproducible and power-efficient fashion against both internal and external disturbances may ensure its reliability for heavy-duty flexible electronics that should endure frequent tension, bending, twisting, and so forth. As a final checkup, we examined whether the SPEEDIN device can function as a reliable and steady heater under repeated tension, bending, and twisting. Figure $5 \mathrm{~b}$ shows the measurement result of the heating temperatures of a 1D-SPEEDIN-on-PI structure at an operating voltage of $2.5 \mathrm{~V}$, after applying $0,10,20$, 30, 50, 75, and 100 times the tension, bending, and twisting in a randomly handled manner as shown in the inset photos. It is apparent that the SPEEDIN device can maintain very stable function under those daily-life occasional disturbances. Additional characterizations involving a standard test for yielding and adhesion and a quantitative bending test with a critical radius of curvature measurement are currently underway.

\section{Conclusions}

In summary, we have developed a facile all-solutionprocessable SPEEDIN methodology that enables continuous single flow-line fabrication of highly durable flexible electronic platforms without resorting to a lithography etching protocol and vacuum processing. By the tactfully controlled procedure of Ag nanoparticle solution coating, baking, and doctor-blading on multidimensional micro- and nanotrench patterns dynamically inscribed in various flexible polymers, the SPEEDIN structure can achieve durably embedded, scratch-proof MNP architectures with comparable optical transmittance as well as electrical conductivity. As one tangible example, we have demonstrated that the SPEEDIN-on-PI structures can work as high-performance flexible heaters with excellent durability and reliability. In-depth, this continuous nanoparticle embedding process can be widely applied to various dielectric materials with superior optical properties for the scalable fabrication of flexible photonic devices $^{52-61}$. Moving forward, SPEEDIN may facilitate further practical applications, including but not limited to protective gear, heavy-duty wearable electronics, and more diverse devices operational under extreme environments involving high temperatures, severe mechanical impacts, and chemical hazards ${ }^{62}$. 


\section{Materials and methods}

\section{SPEEDIN Part I: Fabrication of micro- and nanoscale trench} patterns by dynamic nanoinscribing (DNI)

First, a microridge pattern was fabricated on a 6" Si wafer by $\mathrm{Si}$ deep reactive ion etching. The typical microridge geometry is $\sim 2 \mu \mathrm{m}$ in width, $5 \mu \mathrm{m}$ in height, and $50 \mu \mathrm{m}$ in period (see Fig. 1b) but can be tuned to wider-period microridges, nanoridges, etc., depending on the target trench designs, as actually used for making the samples shown in Fig. S5 in the Supplementary Information. More details on the nanoscale pattern fabrication can be found elsewhere $^{63}$. The DNI mold was then prepared by cleaving the microridge-patterned wafer along the direction perpendicular to the ridge axis, with a typical width of $1-2 \mathrm{~cm}$, which can be extended for scalable DNI processing. The polymer substrates (PC; DE 1-1, CLEAR, Makrofol; PI; HN 1 mil, Kapton) were thoroughly cleaned by isopropyl alcohol (IPA) and deionized water and dried by nitrogen blowing before use. The detailed design, setup, and operation of a custom-built DNI processing system for the fabrication of $1 \mathrm{D}$ - and 2D-micro- and nanopatterns are described elsewhere ${ }^{38,41,46}$. Briefly, the ridge mold edge was mounted on a heater-attached arm and brought to a conformal contact with the substrate with a typical tilting angle of $35-40^{\circ}$. With a mold temperature of $150{ }^{\circ} \mathrm{C}$ for $\mathrm{PC}$ and $310^{\circ} \mathrm{C}$ for PI and a normal contact force of $3 \mathrm{~N}$ maintained, the mold edge slid at a speed of $1 \mathrm{~mm} / \mathrm{s}$ to continuously inscribe the micro- or nanotrench patterns on the substrate. For 2D-DNI, the second stroke was sequentially repeated along the perpendicular direction by using an $\sim 50 \%$ reduced contact force with all the other conditions fixed.

\section{SPEEDIN Part II: Ag nanoparticle solution coating and embedding}

The Ag nanoparticle solution (PS-004, Paru Co., Ltd.; $\sim 100 \mathrm{~nm}$ average diameter) was first spin-coated on the DNI-ed substrate to achieve a specific thickness of $\mathrm{Ag}$ solution for an optimal doctor-blading process, typically at $1000 \mathrm{rpm}$ for $1 \mathrm{~min}$. The Ag solution-coated sample was then soft-baked at $100{ }^{\circ} \mathrm{C}$ for $1 \mathrm{~min}$. A doctor blade typically made of an IPA-soaked fab wipe-wrapped $\mathrm{Si}$ piece edge was used for scraping the soft-baked Ag layer off the substrate surface at the controlled force of $\sim 5 \mathrm{~N}$, which was usually repeated five times. Finally, a hard-baked process at $120^{\circ} \mathrm{C}$ for $5 \mathrm{~min}$ was applied to complete the SPEEDIN structure. The airbrushing of an Ag nanoparticle solution as a control experiment (see Fig. S3 in the Supplementary Information) was conducted by using a custom-designed airbrushing system whose detailed setup and operation procedure were described elsewhere ${ }^{64,65}$.

\section{Characterizations}

All optical photographs were taken by using a digital camera (EOS M5, Canon). SEM imaging was performed by using a field-emission SEM (JSM-6700F, JEOL Ltd.) with an accelerating voltage of $10 \mathrm{kV}$ after sputtering a thin Pt film with 2-3 nm thickness to prevent electron charging. EDX analysis was conducted during SEM imaging by using an Oxford Inca EDX system equipped with the same instrument. The optical transmittance was measured with a wavelength scanning coverage from 200 to $1025 \mathrm{~nm}$ by using a customized spectrometer system (Wonwoo Systems, Co., Ltd.) computer-operated by Ocean Optics software. For characterizing the electrical properties, the silver paste (ELCOAT P-100, CANS Ltd.) was first applied on both ends of the SPEEDIN-ed electrode sample. An electrical current was then recorded by using a digital multimeter (Fluke 15B+, Fluke Corp.). The heating performance was characterized by using IR cameras (FLIR E5 and E6, FLIR Systems, Inc.) under a controlled operation voltage. For preparing the embossed MNP sample for a scratch test, a photoresist microwire pattern (DNR-L300-40 (120 cP), Dongjin SemiChem, Co., Ltd.) was photolithographically defined (Karl Suss MA6) on an identically spin-coated Ag film, followed by wet etching of the exposed Ag (Etchant Type A, Transene Company, Inc.), PR strip (acetone), and IPA rinsing. After putting the sample (either the embossed or SPEEDIN-ed MNP) on a digital scale (Dretec KS-514WT), a tweezer tip was stroked across the microwire line axes while the force and current were read by the scale and DC power supply (Zhaoxin RXN-305D), respectively. The scratch test was sequentially repeated from 1 to $10 \mathrm{~N}$ using one sample. Figure S9 in the Supplementary Information shows photos of the described scratch-proof test. All datapoints presented with error bars in the plots denote the averaged values after repeating measurements three to five times to ensure reliability and accuracy.

\section{Acknowledgements \\ This research was financially supported by the National Research Foundation (NRF) grant (NRF-2015R1A5A1037668) funded by Ministry of Science and ICT of the Korean government. We thank Sunmin Jung and Inho Jang for their assistance during the preliminary experiments. \\ Author details \\ ${ }^{1}$ Department of Mechanical and Automotive Engineering, Seoul National University of Science and Technology, Seoul 01811, Republic of Korea. ${ }^{2}$ Department of Mechanical Engineering, Pohang University of Science and Technology (POSTECH), Pohang 37673, Republic of Korea. ${ }^{3}$ Graduate Program of Energy Technology, School of Integrated Technology, Institute of Integrated Technology, Gwangju Institute of Science and Technology, Gwangju 61005, Republic of Korea. ${ }^{4}$ Department of Mechanical Engineering, Hanbat National University, Daejeon 34158, Republic of Korea. ${ }^{5}$ Department of Chemical Engineering, Pohang University of Science and Technology (POSTECH), Pohang 37673, Republic of Korea. ${ }^{6}$ POSCO-POSTECH-RIST Convergence Research Center for Flat Optics and Metaphotonics, Pohang 37673, Republic of Korea}

\section{Author contributions}

W.L., H.Y. and J.G.O. conceived the principle and designed the research. W.L., H. Chae, and D.K.O. conducted most experiments and characterizations under the supervision of J.R. and J.G.O. M.L., H. Chun, and J.K. contributed to the optical measurement and scratch test. G.Y. and J.P. contributed to the heating 
device application research. W.L., H. Chae, D.K.O., J.R. and J.G.O. wrote the manuscript with all authors' assistance for figure preparation and consistent discussion. All authors read and approved the final manuscript.

\section{Conflict of interest}

The authors declare no competing interests.

Supplementary information The online version contains supplementary material available at https://doi.org/10.1038/s41378-021-00307-5.

Received: 17 March 2021 Revised: 15 July 2021 Accepted: 29 August 2021 Published online: 27 September 2021

\section{References}

1. De Fazio, D. et al. Graphene-quantum dot hybrid photodetectors with low dark-current readout. ACS Nano 14, 11897-11905 (2020).

2. Huo, D. et al. One-dimensional metal nanostructures: from colloidal syntheses to applications. Chem. Rev. 119, 8972-9073 (2019).

3. Singhal, A. V., Charaya, H. \& Lahiri, I. Noble metal decorated graphene-based gas sensors and their fabrication: a review. Crit. Rev. Solid State Mat. Sci. 42 499-526 (2017).

4. Li, W.-J. et al. Integration of metal-organic frameworks into an electrochemical dielectric thin film for electronic applications. Nat. Commun. 7, 11830 (2016).

5. Wang, W., Pfeiffer, P. \& Schmidt-Mende, L. Direct patterning of metal chalcogenide semiconductor materials. Adv. Funct. Mater. 30, 2002685 (2020).

6. Nie, B. et al. Flexible double-sided light-emitting devices based on transparent embedded interdigital electrodes. ACS Appl. Mater. Interfaces 12, 43892-43900 (2020).

7. Wi, J. S. et al. Facile three-dimensional nanoarchitecturing of double-bent gold strips on roll-to-roll nanoimprinted transparent nanogratings for flexible and scalable plasmonic sensors. Nanoscale $\mathbf{9}, 1398-1402$ (2017).

8. Kim, I. et al. Dual-band operating metaholograms with heterogeneous metaatoms in the visible and near-infrared. Adv. Opt. Mater. 9, 2100609 (2021).

9. Yoon, G. et al. Fabrication of three-dimensional suspended, interlayered and hierarchical nanostructures by accuracy-improved electron beam lithography overlay. Sci. Rep. 7, 6668 (2017).

10. Stolt, T. et al. Backward phase-matched second-harmonic generation from stacked metasurfaces. Phys. Rev. Lett. 126, 033901 (2021).

11. Gu, P. et al. Sub-10 nm Au-Ag heterogeneous plasmonic nanogaps. Adv. Mater. Interfaces 7, 1902021 (2020).

12. Cai, J. et al. Highly-facile template-based selective electroless metallization of micro- and nanopatterns for plastic electronics and plasmonics. J. Mater. Chem. C. 7, 4363-4373 (2019).

13. Shakir, S. et al. Fabrication of capillary force induced DNA template Ag nanopatterns for sensitive and selective enzyme-free glucose sensors. Sens. Actuator B Chem. 256, 820-827 (2018).

14. Liu, Z. et al. Three-dimensional self-organization in nanocomposite layered systems by ultrafast laser pulses. ACS Nano 11, 5031-5040 (2017).

15. Lei, Z. et al. Enhanced near-infrared absorption in graphene with multilayer metal-dielectric-metal nanostructure. Opt. Express 24, 20002-20009 (2016).

16. An, C. et al. Plasmonic silver incorporated silver halides for efficient photocatalysis. J. Mater. Chem. A 4, 4336-4352 (2016).

17. Lee, W. et al. Transparent, conformable, active multielectrode array using organic electrochemical transistors. Proc. Natl Acad. Sci. USA 114, 10554 (2017).

18. Lee, S. H. et al. Scalable fabrication of flexible transparent heaters comprising continuously created metallic micromesh patterns incorporated with biomimetic anti-reflection layers. Int. J. Precis Eng. Manuf. Green. Technol. 4, 177-181 (2017).

19. Kim, I. et al. Stimuli-responsive dynamic metaholographic displays with designer liquid crystal modulators. Adv. Mater. 32, 2004664 (2020).

20. Zhao, Z.-J. et al. Nanopattern-embedded micropillar structures for security identification. ACS Appl. Mater. Interfaces 11, 30401-30410 (2019).

21. Lee, D. et al. Polarization-sensitive tunable absorber in visible and near-infrared regimes. Sci. Rep. 8, 12393 (2018).

22. Ok, J. G., Kwak, M. K., Huard, C. M., Youn, H. S. \& Guo, L. J. Photo-roll lithography (PRL) for continuous and scalable patterning with application in flexible electronics. Adv. Mater. 25, 6554-6561 (2013).
23. Ok, J. G. et al. Continuous and scalable fabrication of flexible metamaterial films via roll-to-roll nanoimprint process for broadband plasmonic infrared filters. Appl. Phys. Lett. 101, 4 (2012).

24. Gensch, M. et al. Correlating nanostructure, optical and electronic properties of nanogranular silver layers during polymer-template-assisted sputter deposition. ACS Appl. Mater. Interfaces 11, 29416-29426 (2019).

25. Schwartzkopf, M. et al. Real-time monitoring of morphology and optical properties during sputter deposition for tailoring metal-polymer interfaces ACS Appl. Mater. Interfaces 7, 13547-13556 (2015).

26. Han, S. et al. High-performance solution-processable flexible and transparent conducting electrodes with embedded $\mathrm{Cu}$ mesh. J. Mater. Chem. C. 6, 4389-4395 (2018).

27. Oh, D. K. et al. Top-down nanofabrication approaches toward single-digitnanometer scale structures. J. Mech. Sci. Technol. 35, 837-859 (2021).

28. Lee, D. et al. Multiple-patterning colloidal lithography-implemented scalable manufacturing of heat-tolerant titanium nitride broadband absorbers in the visible to near-infrared. Microsyst. Nanoeng. 7, 14 (2021).

29. Kim, I., Mun, J., Hwang, W., Yang, Y. \& Rho, J. Capillary-force-induced collapse lithography for controlled plasmonic nanogap structures. Microsyst. Nanoeng. 6, 65 (2020).

30. Kim, I. et al. Cascade domino lithography for extreme photon squeezing. Mater. Today 39, 89-97 (2020)

31. Nam, V. B. et al. Laser digital patterning of conductive electrodes using metal oxide nanomaterials. Nano Converg. 7, 23 (2020).

32. Lee, $\mathrm{H}$. et al. Inducing and probing localized excitons in atomically thin semiconductors via tip-enhanced cavity-spectroscopy. Adv. Funct. Mater. 31, 2102893 (2021).

33. Bello, M. \& Shanmugan, S. Achievements in mid and high-temperature selective absorber coatings by physical vapor deposition (PVD) for solar thermal Application—a review. J. Alloy. Compd. 839, 155510 (2020).

34. Chen, Y. et al. Fabrication of polyimide sacrificial layers with inclined sidewalls based on reactive ion etching. AIP Adv. 4, 031328 (2014).

35. Yun, $\mathrm{T}$. et al. $2 \mathrm{D}$ metal chalcogenide nanopatterns by block copolymer lithography. Adv. Funct. Mater. 28, 1804508 (2018).

36. Ammosova, L., Jiang, Y., Suvanto, M. \& Pakkanen, T. A. Precise micropatterning of silver nanoparticles on plastic substrates. Appl. Surf. Sci. 401, 353-361 (2017).

37. Zhao, Z-J. et al. Effects of polymer surface energy on morphology and properties of silver nanowire fabricated via nanoimprint and E-beam evaporation. Appl. Surf. Sci. 420, 429-438 (2017).

38. Oh, D. K. et al. Facile and scalable fabrication of flexible reattachable ionomer nanopatterns by continuous multidimensional nanoinscribing and lowtemperature roll imprinting. ACS Appl. Mater. Interfaces 11, 12070-12076 (2019).

39. Zhang, C. et al. Printed photonic elements: nanoimprinting and beyond. J. Mater. Chem. C. 4, 5133-5153 (2016).

40. Ok, J. G., Shin, Y. J., Park, H. J. \& Guo, L. J. A step toward next-generation nanoimprint lithography: extending productivity and applicability. Appl. Phys. A Mater. Sci. Process. 121, 343-356 (2015).

41. Ok, J. G., Panday, A., Lee, T. \& Jay Guo, L. Continuous fabrication of scalable 2-dimensional (2D) micro- and nanostructures by sequential 1D mechanical patterning processes. Nanoscale 6, 14636-14642 (2014).

42. Chen, Q. et al. Layer-by-layer spray-coating of cellulose nanofibrils and silver nanoparticles for hydrophilic interfaces. ACS Appl. Nano Mater. 4, 503-513 (2021).

43. Glier, T. E. et al. Functional printing of conductive silver-nanowire photopolymer composites. Sci. Rep. 9, 6465 (2019).

44. Oh, Y. S. et al. Temperature-controlled direct imprinting of Ag ionic ink: flexible metal grid transparent conductors with enhanced electromechanical durability. Sci. Rep. 7, 11220 (2017).

45. Youn, H., Lee, T. \& Guo, L. J. Multi-film roll transferring (MRT) process using highly conductive and solution-processed silver solution for fully solutionprocessed polymer solar cells. Energy Environ. Sci. 7, 2764-2770 (2014).

46. Oh, D. K. et al. Tailored nanopatterning by controlled continuous nanoinscribing with tunable shape, depth, and dimension. ACS Nano 13, 11194-11202 (2019).

47. Ok, J. G., Ahn, S. H., Kwak, M. K. \& Guo, L. J. Continuous and high-throughput nanopatterning methodologies based on mechanical deformation. J. Mater. Chem. C. 1, 7681-7691 (2013).

48. Beibei, Z., Zakya, H. K. \& Filbert, J. B. Transparent electrodes based on twodimensional Ag nanogrids and double one-dimensional Ag nanogratings for organic photovoltaics. J. Photonics Energy 5, 1-13 (2014). 
49. Catrysse, P. B. \& Fan, S. Nanopatterned metallic films for use as transparent conductive electrodes in optoelectronic devices. Nano Lett. 10, 2944-2949 (2010).

50. Hong, R., Shao, W., Ji, J., Tao, C. \& Zhang, D. Thermal annealing induced the tunable optical properties of silver thin films with linear variable thickness. Superlattices Microstruct. 118, 170-176 (2018).

51. Ahn, B. Y. \& Lewis, J. A. Amphiphilic silver particles for conductive inks with controlled wetting behavior. Mater. Chem. Phys. 148, 686-691 (2014).

52. Yoon, G. et al. Printable nanocomposite metalens for high-contrast nearinfrared imaging. ACS Nano 15, 698-706 (2021).

53. Yoon, G., Kim, K., Huh, D., Lee, H. \& Rho, J. Single-step manufacturing of hierarchical dielectric metalens in the visible. Nat. Commun. 11, 2268 (2020).

54. Chen, Y., Ai, B. \& Wong, Z. J. Soft optical metamaterials. Nano Converg. 7, 18 (2020).

55. Kim, K., Yoon, G., Baek, S., Rho, J. \& Lee, H. Facile nanocasting of dielectric metasurfaces with sub-100 nm resolution. ACS Appl. Mater. Interfaces 11, 26109-26115 (2019).

56. Yang, Y. et al. Revealing structural disorder in hydrogenated amorphous silicon for a low-loss photonic platform at visible frequencies. Adv. Mater. 33, 2005893 (2021).

57. Kim, I. et al. Holographic metasurface gas sensors for instantaneous visual alarms. Sci. Adv. 7, eabe9943 (2021).
58. Kim, I. et al. Pixelated bifunctional metasurface-driven dynamic vectorial holographic color prints for photonic security platform. Nat. Commun. 12 3614 (2021).

59. Kim, J. et al. Geometric and physical configurations of meta-atoms for advanced metasurface holography. InfoMat 3, 739-754 (2021).

60. Yoon, G., Tanaka, T., Zentgraf, T. \& Rho, J. Recent progress on metasurfaces: applications and fabrication. J. Phys. D Appl. Phys. 54, 383002 (2021).

61. Jung, W. et al. Three-dimensional nanoprinting via charged aerosol jets. Nature 592, 54-59 (2021)

62. Jo, H. S., An, S., Kwon, H.-J., Yarin, A. L. \& Yoon, S. S. Transparent bodyattachable multifunctional pressure, thermal, and proximity sensor and heater. Sci. Rep. 10, 2701 (2020).

63. Ok, J. G. et al. Continuous patterning of nanogratings by nanochannel-guided lithography on liquid resists. Adv. Mater. 23, $4444-4448$ (2011).

64. Chun, $\mathrm{H}$. et al. Facile airbrush fabrication of gas diffusion layers comprising fine-patterned hydrophobic double-layer and hydrophilic channel for improved water removal in polymer electrolyte membrane fuel cells. Int. J. Precis. Eng. Manuf. Green Technol. https://doi.org/10.1007/s40684-020-00254-y $\% 20$ (2020).

65. Koo, S. et al. Controlled airbrush coating of polymer resists in Roll-to-Roll nanoimprinting with regimented residual layer thickness. Int. J. Precis. Eng. Manuf. 17, 943-947 (2016). 